\title{
Researched on Initiation Synchronization of Addressable Initiation System
}

\author{
Guofu Yin ${ }^{1, \mathrm{a}}$, Liming $\mathrm{Li}^{1}$ and Qiao $\mathrm{Lu}^{1, \mathrm{~b}}$ \\ ${ }^{1}$ Shanxi Applied physic-Chemistry Research Institute, State Key Laboratory of Applied physic-Chemistry Research, Xi'an of China \\ ayinguofu521@126.com, ,chufengde01@163.com
}

\begin{abstract}
Addressable initiation system was established by bus topology structure and information coding which owns the ability to safely and reliably control up to 256 initiators on a signal bus according to the communication protocol which is a robust two-way command response type protocol. Also, the two-way digital communication was achieved between intelligent bus controller and smart initiator by multiplexed serial data bus. The synchronous performance of addressable initiation system was researched. The result shows that each smart initiator will performance the built-in logic control, and arm electronic safe switch after receiving the synchronous signal sent by intelligent bus controller. Then they discharged the electrical energy of the firing capacitor as approximately synchronous, and were initiated synchronously at $4 \pm 0.2 \mu \mathrm{s}$. It indicates that this initiation system has favorable synchronization.
\end{abstract}

Keywords. Addressable initiation system, smart initiator, communication protocol, semiconductor bridge.

\section{Introduction}

Initiation system as the control center of weapons and ammunition system, responsible for safeguarding the warhead-war and wartime timely and reliable security arming and detonation operations, etc. With the developing of weapons systems toward miniaturization, low power, high accuracy, high damage, the addressing detonation initiation system is emerging which revolutionary transforms the initiation system from the typical centre firing control to the centralized control and distributed initiation [1].In paper, the addressable initiation system was established by bus topology structure and information coding. Also, the two-way communication was achieved between intelligent bus controller and smart initiator by multiplexed serial data bus. Intelligent bus controller can centralized control every smart initiators on bus and achieve multi-mode initiation of smart initiator. Also, the initiation synchronization, which is very important in weapons systems such as missile warhead, spacecraft solar panels unfold, solid rocket motor ignition, has been researched of the system.

\section{Addressable Initiation System Design}

The addressable initiation system was designed, and shown in Figure 1, which consists of intelligent bus controller, data bus, a number of smart initiators and communication protocols [2,3]. Intelligent bus controller can send identity address (ID) and instruction to all smart initiator on the initiation bus [4,5]. As each smart initiator received an ID, they will compare the ID with their own ID store in ROM. If the received ID of each smart initiator is entirely same with self ID, it will execute pre-set instruction, and release energy store in capacitance to fire the energy device such as 
semiconductor bridge (SCB), hot wire and bridge film which will result to fire charge sequence, and output strong explosion waves. If two ID is completely different, the smart initiator will stop all the action and wait for next ID and instruction which are sent by intelligent bus controller until two ID are completely match each other. The system is an electronically addressable, low power ordnance initiation system, consisting of a controller, smart initiators, and wire ham.

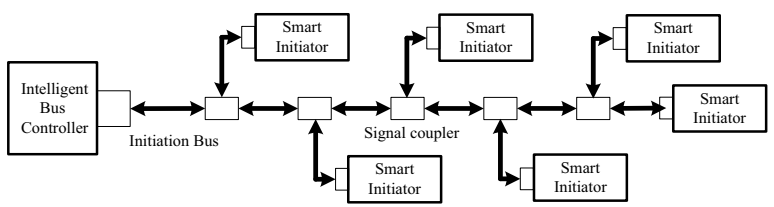

Fig. 1. Schematic of addressable initiation system

Intelligent bus controller as a initiation system central nervous system, centralized control the all action of the system. Shown in figure 2, it was designed including two energy regulation circuit, micro-control circuit, communication circuit and electronic Safe/Arm circuit. It receives instruction and energy from guidance and control unit, also control the instruction and the energy to reach every specified initiator. And then, it will receive the feedback information of the smart initiator, and continue to feedback the results of the implementation of the directive to the guidance and control unit.

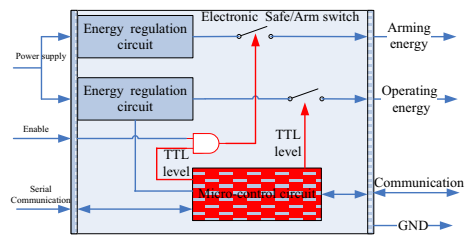

Fig.2. schematic of intelligent bus controller

Smart initiator was designed by using microelectronic integrated design technology. Shown in figure 3 and figure 4, some function circuits were integrated within it including anti-electromagnetic interference and (EMI) Static electricity (ESD)circuit, information identifying circuit, micro-control circuit, electronic safe/arm circuit, energy storage circuit, fire circuit and semiconductor bridge.

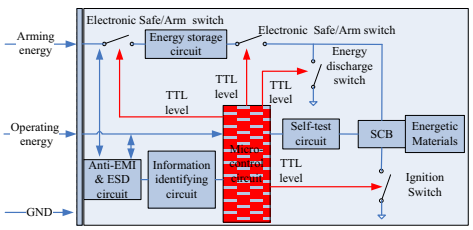

Fig.3. schematic of smart initiator

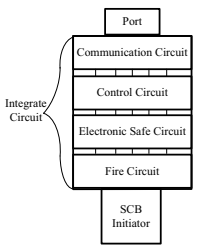

Fig.4. Integrate smart Initiator

Each smart initiator has a unique address code ID stored in micro-control circuit ROM. It can be ID to finish the safe/arm and fire function. Only receive Instruction signals consistent with their ID, smart initiator will carry out the 
initiation function. And other spurious signals will be rejected by the information identifying circuit in order to achieve a rapid addressing of the initiator. In addition, the anti-electromagnetic electronic circuit was designed in smart initiator, so that the information identifying circuit with anti-interference ability which can prevent accidental initiation caused the error identification. It will improve the safety and reliability of smart initiator. Static electronic is great harm for electrical explosion devices (EED), and must design antistatic electronic circuit in smart initiator. Due to the different characteristics of antistatic with each circuit, so two-stage anti-static circuit was designed. Information identifying circuit and logic control circuit is protected by semiconductor discharge transistors and TVS diodes. And then semiconductor bridge and electronic switch is protected by the CMOSFET which is sensitive to electrostatic. In addition, between the information identify circuit and logic control circuit designed the magnetic isolation circuit. Even in the case of a faulty initiator in system, it does not result in paralysis of the entire bus network.

\section{Experiment and Analyses}

The synchronization of initiation system is harsh demanding in the weapons systems such as missile warhead, spacecraft solar panels unfold, solid rocket motor ignition and other weapons systems. Therefore the synchronous performance of addressing detonation initiation system will be developed in this paper. Experiments using three-point addressable initiation system, three channel current loop use for testing the current characteristics of firing capacitor discharge, three channel oscilloscope probe use for test the voltage of across the semiconductor bridge chip, and three channel DET10A photo-detectors was be used to test the initiation output performance of SCB initiator. Experiment principle as shown, including the intelligent bus controller, data bus, signal couplers, three smart initiators, oscilloscope, current loop and photo-detectors. Each smart initiator integrates a $47 \mu \mathrm{F}$ firing capacitor and it charge voltage is $15 \mathrm{~V}$.

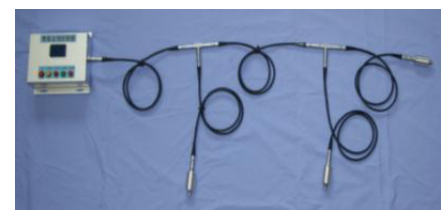

Fig.5. Three-point initiation system

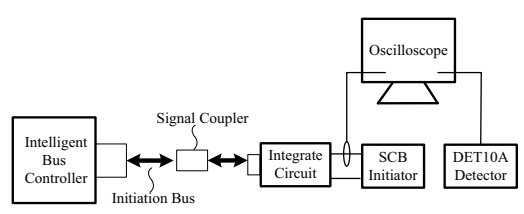

Fig.6. Schematics of firing test

(1) Synchronization of firing current and voltage

Firing current I reflect the changes of SCB resistor. As shown in Fig.7, firing current of three-point SCB energy element of smart initiator are gradually increased from 0 , and reach a maximum $6 \pm 0.5 \mathrm{~A}$ at $4 \pm 0.2 \mu \mathrm{s}$, and at that time each smart initiator was initiated. It indicates that firing current of three-point initiators are approximately synchronous.

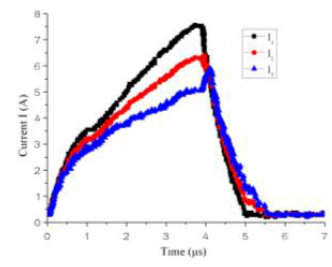

Fig.7. Graph of firing current characteristic 


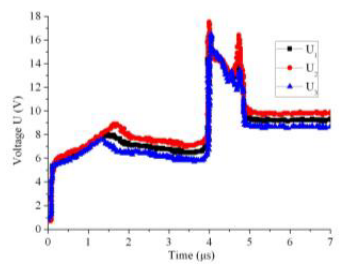

Fig.8. Graph of firing voltage characteristic

The voltage characteristic of across the SCB energy elements is shown in Fig.8. At the A-B stage, the SCB is heated by the passing current, and the voltage reaches its first peak at the point $\mathrm{B}$. At the B-C stage, owing to ohmic heating, the silicon in SCB temperature increases, and solid silicon melts and transforms to liquid silicon. At the C-D, liquid silicon evaporates and transforms to gaseous silicon. At the D-E stage, the rapidly growing voltage synchronously reaches the maximum duration the $4 \pm 0.2 \mu \mathrm{s}$, and initiates a discharge in gaseous silicon and plasma formation. It indicated the firing voltage of across the SCB of three-point smart initiators are approximatively synchronous.

(2)Output synchronization

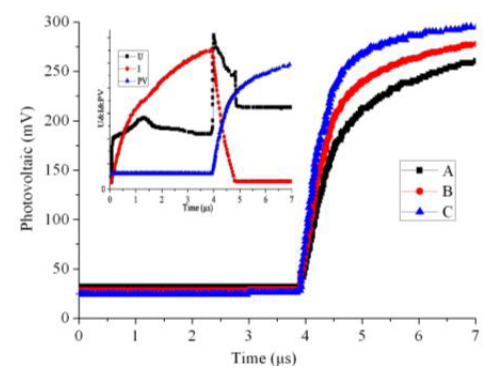

Fig.9. Graph of optical-eletronic detector output

DET10A photo-detectors were used to detect the initiation output of smart initiator. Fig.9 shows that the three-point smart initiators were initiated at $4 \pm 0.2 \mu \mathrm{s}$ when, and the initiation output ability of each smart initiator reached the maximum at $7 \mu \mathrm{s}$. It indacates that the output of three-point smart initiators are approximately synchronous.

\section{Summary}

The synchronization of initiation system is harsh demanding in the weapons systems such as missile warhead, spacecraft solar panels unfold, solid rocket motor ignition and other weapons systems. Therefore the addressable initiation system was designed and this system has demonstrated the ability to arm and fire 256 initiators on a single bus. Also the synchronous performance of addressable initiation system was researched. The result shows that each smart initiator will performance the built-in logic control, and arm electronic safe switch after receiving the synchronous signal sent by intelligent bus controller. Then they discharged the electrical energy of the firing capacitor as approximatively synchronous, and were initiated synchronously at $4 \pm 0.2 \mu$ s. It indicates that this initiation system has favorable synchronization.

\section{References}

1. David B. Novotney and Andrew Kochanek, Ensign-Bickford Aerospace \& Defense Company, Simsbury, CT, 06070, Intelligent Initiation Systems for Divert and Attitude Control Applications, AIAA 2005- 4502

2. Steven D. Nelson, Redondo Beach, CA. Networked electronic ordnance system, US7752970B2, 13(2010) 
3. D.L. WANG, B.Y. GUO, S.K. NING, Design and implementation of explosive network of electronic detonators and communication protocol based on the RS-485, Joumal of Shanxi University of Technology (Natural Sicence Edition), 26, 1(2010)

4. J.Y. WANG, L.M. LI, G.F. YIN, et al., Design on Intelligent Initiation System Based on RS-485 Bus, Initiators \& Pyrotechnics, 2 (2013)

5. T. LIU, Y.Z. FU, K.N. XIE, et al., Design and verification of new kind of integrated ordance initiation control system, Computer Science, 35,4(2008) 